\title{
Peristaltic Motion of Non-Newtonian Fluid with Heat and Mass Transfer through a Porous Medium in Channel under Uniform Magnetic Field
}

\author{
Nabil T. M. Eldabe, ${ }^{1}$ Bothaina M. Agoor, ${ }^{2}$ and Heba Alame ${ }^{2}$ \\ ${ }^{1}$ Department of Mathematics, Faculty of Education, Ain Shams University, Cairo 11566, Egypt \\ ${ }^{2}$ Department of Mathematics, Faculty of Science, Fayoum University, P.O. Box 63514, Fayoum, Egypt \\ Correspondence should be addressed to Bothaina M. Agoor; bma00@fayoum.edu.eg
}

Received 10 January 2014; Accepted 15 March 2014; Published 10 April 2014

Academic Editor: Kuo-Kang Liu

Copyright ( $\odot 2014$ Nabil T. M. Eldabe et al. This is an open access article distributed under the Creative Commons Attribution License, which permits unrestricted use, distribution, and reproduction in any medium, provided the original work is properly cited.

This paper is devoted to the study of the peristaltic motion of non-Newtonian fluid with heat and mass transfer through a porous medium in the channel under the effect of magnetic field. A modified Casson non-Newtonian constitutive model is employed for the transport fluid. A perturbation series' method of solution of the stream function is discussed. The effects of various parameters of interest such as the magnetic parameter, Casson parameter, and permeability parameter on the velocity, pressure rise, temperature, and concentration are discussed and illustrated graphically through a set of figures.

\section{Introduction}

Peristaltic motion is a phenomenon that occurs when expansion and contraction of an extensible tube in a fluid generate progressive waves which propagate along the length of the tube, mixing and transporting the fluid in the direction of wave propagation. In some biomedical instruments, such as heart-lung machines, peristaltic motion is used to pump blood and other biological fluids [1]. Peristaltic pumping is a form of fluid transport generally from a region of lower to higher pressure, by means of a progressive wave of area contraction or expansion, which propagates along the length of a tube like structure. Some electrochemical reactions are held responsible for this phenomenon. This mechanism occurs in swallowing of food through oesophagus, in the ureter, the gastro intestinal tract, the bile duct, and even in small blood vessels. It has now been accepted that most of the physiological fluids behave like a non-Newtonian fluids. The peristaltic flows have attracted a number of researchers because of wide applications in physiology and industry. The theoretical work of peristaltic transport primarily with the inertia free Newtonian flow driven by a sinusoidal transverse wave of small amplitude is investigated by Fung et al. [2]. Burns and Parkes [3] studied the peristaltic motion of a viscous fluid through a pipe and channel by considering sinusoidal variations at the walls. A mathematical study of the peristaltic transport of Casson fluid is given by Mernone and Mazumdar $[4,5]$; they used the perturbation method to solve the problem. Mekheimer [6,7] studied the peristaltic transport of MHD flow. Peristaltic transport of Casson fluid in a channel is discussed by Nagarani and Sarojamma [8, 9]. El Shehawy et al. [10] Studied the peristaltic transport in a symmetric channel through a porous medium. Finite element solutions for non-Newtonian pulsatile flow in a nonDarcian porous medium are given by Bharagava et al. [11]. Mekheirmer and Abd elmaboud [12] discussed the influence of heat transfer and magnetic field on peristaltic transport. Nadeem et al. [13] have discussed the influence of heat and mass transfer on peristaltic flow of third order fluid in a diverging tube. Abdelmaboud and Mekheimer [14] analyzed the transport of second order fluid through a porous medium. Abd Elmaboud [15] studied the heat transfer characteristics of micropolar fluid through an isotropic porous medium in a two-dimensional channel with rhythmically contracting 
walls. El-dabe et al. [16] studied the effects of radiation on the unsteady flow of an incompressible non-Newtonian (Jeffrey) fluid through porous medium. Mustafa et al. [17] studied the peristaltic transport of nanofluid in a channel with complaint walls. Anwr Beg and Tripathi [18] introduced a theoretical study to examine the peristaltic pumping with doublediffusive convection in nanofluids through a deformable channel. El-dabe et al. [19] have discussed the effects of heat and mass transfer on the MHD flow of an incompressible, electrically conducting couple stress fluid through a porous medium in an asymmetric flexible channel over which a traveling wave of contraction and expansion is produced, resulting in a peristaltic motion. El-dabe et al. [20] studied the peristaltic motion of incompressible micropolar fluid through a porous medium in a two-dimensional channel under the effects of heat absorption and chemical reaction in the presence of magnetic field. Ebaid and Emad Aly [21] showed the mathematical model describing the slip peristaltic flow of nanofluid application to the cancer treatment. Emad and Ebaid [22] applied two different analytical and numerical methods to solve the system describing the mixed convection boundary layer nanofluids flow along an inclined plate embedded in a porous medium. Abd Elmaboud [23] investigated the magneto thermodynamic aspects micropolar fluid (blood model) through an isotropic porous medium in a nonuniform channel with rhythmically contracting walls. Noreen et al. [24] studied the mathematical model to investigate the mixed convective heat and mass transfer effects on peristaltic flow of magnetohydrodynamic pseudoplastic fluid in a symmetric channel. Hayat et al. [25] discussed the effects of heat and mass transfer on the peristaltic flow in the presence of an induced magnetic field. Noreen [26] consider the peristaltic flow of third order nanofluid in an asymmetric channel with an induced magnetic field.

The main aim of this work is to study the peristaltic motion of non-Newtonian fluid with heat and mass transfer through a porous medium in the channel under the effect of magnetic field. A modified Casson non-Newtonian constitutive model is employed for the transport fluid. A perturbation series' method of solution of the stream function is discussed. The effects of various parameters of interest such as the magnetic parameter, Casson parameter, and permeability parameter on the velocity, pressure rise, and temperature are discussed and illustrated graphically through a set of figures.

\section{Mathematical Analysis}

Consider the peristaltic motion of non-Newtonian fluid through a porous medium in two-dimensional channel, having width $d$. A rectangular coordinate system $x, y$ is chosen such that $x$-axis lies along the direction of wave progression and $y$-axis normal to it. The fluid is subjected to a constant magnetic field $\underline{B}=\left(0, B_{0}, 0\right)$. Let $u$ and $v$ be the velocity components. The vertical displacements for the upper and lower walls are $\zeta$ and $-\zeta$, see Figure 1 , where $\zeta$ is defined by

$$
\zeta(x, t)=a \cos \frac{2 \pi}{\lambda}(x-c t) .
$$

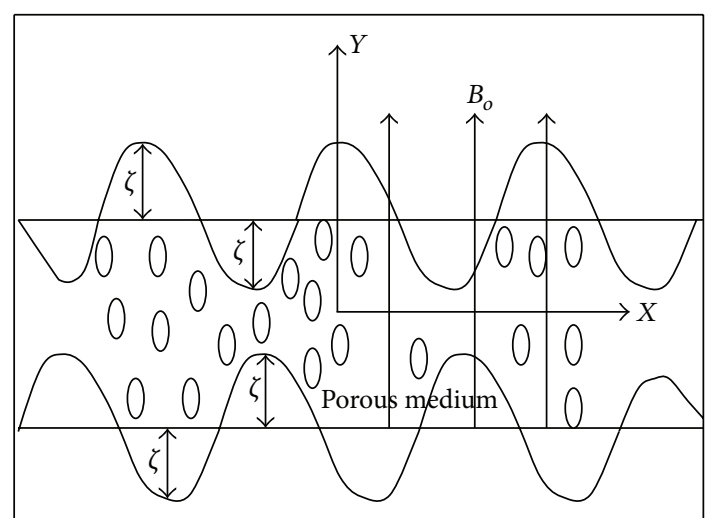

FIGURE 1: Sketch of the problem.

$\lambda$ is the wavelength, $t$ is the time and $a$ is the amplitude of the sinusoidal waves travelling along the channel at velocity $c$. The constitutive equation for the non-Newtonian Casson fluid can be written as in [27].

Consider

$$
\tau_{i j}= \begin{cases}2\left(\mu_{B}+\frac{p_{y}}{\sqrt{2 \pi}}\right) e_{i j}, & \pi>\pi_{c}, \\ 2\left(\mu_{B}+\frac{P_{y}}{\sqrt{2 \pi_{c}}}\right) e_{i j}, & \pi<\pi_{c},\end{cases}
$$

where $\tau_{i j}$ is the components of the stress tensor, $\pi=e_{i j} e_{j i}$ and $e_{i j}$ are the $(i, j)$ th components of the deformation rate, $\pi$ is the product of the component of deformation rate by itself, $\pi_{c}$ is a critical value of this product based on the NakamuraSawada model [27], $\mu_{B}$ is the plastic dynamic viscosity of the non-Newtonian fluid, and $p_{y}$ is yield stress of slurry fluid.

The equations governing the fluid motion can be written as follows.

The continuity equation is

$$
\frac{\partial u}{\partial x}+\frac{\partial v}{\partial y}=0
$$

The momentum equations are

$$
\begin{aligned}
& \rho\left(\frac{\partial u}{\partial t}\right.\left.+u \frac{\partial u}{\partial x}+v \frac{\partial u}{\partial y}\right) \\
&=-\frac{\partial P}{\partial x}+\left(\mu+\mu_{B}\right)\left(\frac{\partial^{2} u}{\partial x^{2}}+\frac{\partial^{2} u}{\partial y^{2}}\right)-\left(\sigma B_{0}^{2}+\frac{\mu}{k}\right) u \\
& \rho\left(\frac{\partial v}{\partial t}+u \frac{\partial v}{\partial x}+v \frac{\partial v}{\partial y}\right) \\
&=-\frac{\partial P}{\partial y}+\left(\mu+\mu_{B}\right)\left(\frac{\partial^{2} v}{\partial x^{2}}+\frac{\partial^{2} v}{\partial y^{2}}\right)-\left(\sigma B_{0}^{2}+\frac{\mu}{k}\right) v .
\end{aligned}
$$

The energy equation is

$$
\left(\frac{\partial T}{\partial t}+u \frac{\partial T}{\partial x}+v \frac{\partial T}{\partial y}\right)=\frac{k_{1}}{\rho c_{p}}\left(\frac{\partial^{2} T}{\partial x^{2}}+\frac{\partial^{2} T}{\partial y^{2}}\right)
$$


The concentration equation is

$$
\left(\frac{\partial C}{\partial t}+u \frac{\partial C}{\partial x}+v \frac{\partial C}{\partial y}\right)=D_{m}\left(\frac{\partial^{2} C}{\partial x^{2}}+\frac{\partial^{2} C}{\partial y^{2}}\right)
$$

Lorentz force:

$$
\underline{F}=\underline{J} \wedge \underline{B}, \quad \underline{J}=\sigma \underline{V} \wedge \underline{B}
$$

where $k$ is the permeability of the medium, $J$ is the current density, $c_{p}$ is the specific heat of the fluid, $k_{1}$ is the coefficient of heat conduction, $T$ is the temperature of the fluid, $D_{m}$ is the coefficient of mass diffusivity, $C$ is the concentration of the fluid, $\mu$ is the coefficient of viscosity, $\sigma$ is the electrical conductivity, $P$ is pressure, and $B_{o}$ is the strength of the applied magnetic field.

The appropriate boundary conditions are

$$
\begin{array}{r}
u=0, \quad v=\frac{\partial \zeta}{\partial t}, \quad T=T_{1}, \quad C=C_{1} \\
\text { at } y=d+\zeta, \\
u=0, \quad v=-\frac{\partial \zeta}{\partial t}, \quad T=T_{0}, \quad C=C_{0} \\
\text { at } y=-d-\zeta .
\end{array}
$$

Using the following nondimensional variables:

$$
\begin{gathered}
x^{\prime}=\frac{x}{d}, \quad y^{\prime}=\frac{y}{d}, \quad u^{\prime}=\frac{u}{c}, \quad v^{\prime}=\frac{v}{c}, \quad t^{\prime}=\frac{c t}{d}, \\
p^{\prime}=\frac{p}{\rho c^{2}}, \quad \zeta^{\prime}=\frac{\zeta}{d}, \quad \varepsilon=\frac{a}{d}, \\
T=\left(T_{1}-T_{0}\right) \theta+T_{0}, \quad C=\left(C_{1}-C_{0}\right) \phi+C_{0},
\end{gathered}
$$

equations (4)-(8) after dropping the stars mark reduce to

$$
\begin{aligned}
\left(\frac{\partial u}{\partial t}+\right. & \left.u \frac{\partial u}{\partial x}+v \frac{\partial u}{\partial y}\right) \\
& =-\frac{\partial P}{\partial x}+\left(\tau_{0}+\frac{1}{R_{e}}\right)\left(\frac{\partial^{2} u}{\partial x^{2}}+\frac{\partial^{2} u}{\partial y^{2}}\right)-\left(M+\frac{1}{K}\right) u \\
\left(\frac{\partial v}{\partial t}+\right. & \left.u \frac{\partial v}{\partial x}+v \frac{\partial v}{\partial y}\right) \\
& =-\frac{\partial P}{\partial y}+\left(\tau_{0}+\frac{1}{R_{e}}\right)\left(\frac{\partial^{2} v}{\partial x^{2}}+\frac{\partial^{2} v}{\partial y^{2}}\right)-\left(M+\frac{1}{K}\right) v \\
& \left(\frac{\partial \theta}{\partial t}+u \frac{\partial \theta}{\partial x}+v \frac{\partial \theta}{\partial y}\right)=\frac{k_{1}}{\rho c_{p}}\left(\frac{\partial^{2} \theta}{\partial x^{2}}+\frac{\partial^{2} \theta}{\partial y^{2}}\right) \\
& \left(\frac{\partial \phi}{\partial t}+u \frac{\partial \phi}{\partial x}+v \frac{\partial \phi}{\partial y}\right)=\frac{k_{1}}{\rho c_{p}}\left(\frac{\partial^{2} \phi}{\partial x^{2}}+\frac{\partial^{2} \phi}{\partial y^{2}}\right)
\end{aligned}
$$

with the boundary conditions

$$
\begin{array}{r}
u=0, \quad v=\alpha \varepsilon \sin \alpha(x-t), \quad \theta=1, \quad \phi=1 \\
\text { at } y=1+\zeta, \\
u=0, \quad v=-\alpha \varepsilon \sin \alpha(x-t), \quad \theta=0, \quad \phi=0 \\
\text { at } y=-1-\zeta,
\end{array}
$$

where $M=\sigma B_{0}^{2} d / \rho c$ is the magnetic parameter, $K=c k / \vartheta d$ is the permeability parameter, $\alpha=2 d \pi / \mu$ is the wave number, $\tau_{0}=\mu_{B} / \rho c d$ is the Casson parameter, and $R_{e}=\rho c d / \mu$ is the Reynolds number.

Now, we shall define a stream function $\psi$ as $u=\psi_{y}$ and $v=-\psi_{x}$ then $(10)$ can be written as

$$
\begin{aligned}
\psi_{y t}+\psi_{y} \psi_{y x}-\psi_{x} \psi_{y y} \\
=-\frac{\partial p}{\partial x}+\left(\tau_{0}+\frac{1}{R_{e}}\right)\left(\psi_{y y y}+\psi_{y x x}\right)-\left(M+\frac{1}{K}\right) \psi_{y}, \\
-\psi_{x t}-\psi_{y} \psi_{x x}+\psi_{x} \psi_{x y} \\
=-\frac{\partial p}{\partial y}-\left(\tau_{0}+\frac{1}{R_{e}}\right)\left(\psi_{x x x}+\psi_{y y x}\right)+\left(M+\frac{1}{K}\right) \psi_{x} \\
\left(\frac{\partial \theta}{\partial t}+\psi_{Y} \frac{\partial \theta}{\partial x}-\psi_{X} \frac{\partial \theta}{\partial y}\right)=\frac{k_{1}}{\rho c_{p}}\left(\frac{\partial^{2} \theta}{\partial x^{2}}+\frac{\partial^{2} \theta}{\partial y^{2}}\right) \\
\left(\frac{\partial \phi}{\partial t}+\psi_{Y} \frac{\partial \phi}{\partial x}-\psi_{X} \frac{\partial \phi}{\partial y}\right)=D_{m}\left(\frac{\partial^{2} \phi}{\partial x^{2}}+\frac{\partial^{2} \phi}{\partial y^{2}}\right)
\end{aligned}
$$

with conditions

$$
\begin{array}{r}
\psi_{y}=0, \quad \psi_{x}=-\alpha \varepsilon \sin \alpha(x-t), \quad \theta=1, \quad \phi=1 \\
\text { at } y=1+\zeta, \\
\psi_{y}=0, \quad \psi_{x}=\alpha \varepsilon \sin \alpha(x-t), \quad \theta=0, \quad \phi=0 \\
\text { at } y=-1-\zeta .
\end{array}
$$

Express a stream function $\psi, p, \theta$, and $\phi$ as a series in terms of small amplitude ratio $\varepsilon$, we have

$$
\begin{gathered}
\psi(x, y, t)=\psi_{0}+\varepsilon \psi_{1}+\cdots, \\
p(x, y)=p_{0}+\varepsilon p_{1}+\cdots, \\
\theta(x, y, t)=\theta_{0}+\varepsilon \theta_{1}+\cdots, \\
\phi(x, y, t)=\phi_{0}+\varepsilon \phi_{1}+\cdots,
\end{gathered}
$$

where $\psi_{0}$ is a function of $y$ only. Substituting (14) in (12) and collecting the terms in $\varepsilon$, we get the following system of equations. 
Coefficient of $\varepsilon^{0}$ :

$\frac{\partial p_{0}}{\partial y}=0$, this leads to $P_{o}$ is a function of $x, p_{0}=c_{1}(x)$,

$$
\begin{gathered}
\psi_{\text {oyyy }}-\lambda_{1}^{2} \psi_{0 y}+L=0, \\
\frac{\partial^{2} \theta_{0}}{\partial y^{2}}=0 \\
\frac{\partial^{2} \phi_{0}}{\partial y^{2}}=0
\end{gathered}
$$

where

$$
\lambda_{1}=\sqrt{\frac{(M+(1 / K))}{\left(\tau_{0}+\left(1 / R_{e}\right)\right)}}, \quad L=\left(\frac{\partial p_{0} / \partial x}{\left(\tau_{0}+\left(1 / R_{e}\right)\right)}\right) .
$$

and coefficient of $\varepsilon$ :

$$
\begin{gathered}
\psi_{1 y t}+\psi_{0 y} \psi_{1 y x}-\psi_{1 x} \psi_{0 y y} \\
=-\frac{\partial p_{1}}{\partial x}+\left(\tau_{0}+\frac{1}{R_{e}}\right)\left(\psi_{1 y y y}+\psi_{1 y x x}\right)-\left(M+\frac{1}{K}\right) \psi_{1 y} \\
-\psi_{1 x t}-\psi_{0 y} \psi_{1 x x} \\
=-\frac{\partial p_{1}}{\partial y}-\left(\tau_{0}+\frac{1}{R_{e}}\right)\left(\psi_{1 x x x}+\psi_{1 y y x}\right)+\left(M+\frac{1}{K}\right) \psi_{1 x}, \\
\left(\frac{\partial \theta_{1}}{\partial t}+\psi_{0 y} \frac{\partial \theta_{1}}{\partial x}-\psi_{1 x} \frac{\partial \theta_{1}}{\partial y}\right)=\frac{k_{1}}{\rho c_{p}}\left(\frac{\partial^{2} \theta_{1}}{\partial x^{2}}+\frac{\partial^{2} \theta_{1}}{\partial y^{2}}\right), \\
\left(\frac{\partial \phi_{1}}{\partial t}+\psi_{0 y} \frac{\partial \phi_{1}}{\partial x}-\psi_{1 x} \frac{\partial \phi_{1}}{\partial y}\right)=D_{m}\left(\frac{\partial^{2} \phi_{1}}{\partial x^{2}}+\frac{\partial^{2} \phi_{1}}{\partial y^{2}}\right) .
\end{gathered}
$$

Also, boundary conditions (13) can be written after using the Taylor series expansions about $y= \pm 1 \pm \zeta$ as follows:

$$
\begin{gathered}
\psi_{0 y}(1)=0, \quad \theta_{0}(1)=1, \quad \phi_{0}(1)=1, \\
\psi_{1 y}(1)=-\psi_{0 y y}(1) \cos \alpha(x-t), \\
\theta_{1}(1)=-\theta_{0 y}(1) \cos \alpha(x-t), \\
\psi_{0}(-1)=0, \quad \theta_{0}(-1)=0, \quad \phi_{0}(-1)=0, \\
\psi_{1 x}(1)=-\alpha \sin \alpha(x-t), \\
\psi_{0 y}(-1)=0, \quad \phi_{1}(1)=-\phi_{0 y}(1) \cos \alpha(x-t) .
\end{gathered}
$$

Using conditions (24) with (15), the solution of (16) can be written as

$$
\psi_{0}(y)=\frac{-L}{\lambda_{1}}\left(y-\frac{\sinh \lambda_{1} y}{\lambda_{1} \cosh \lambda_{1}}\right)
$$

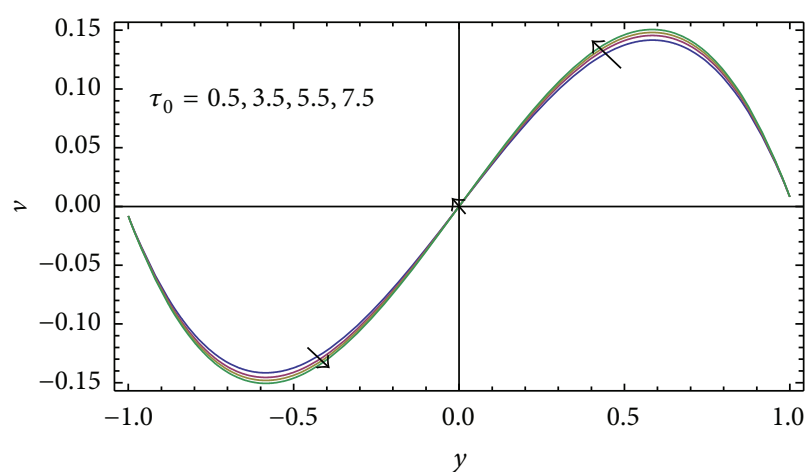

FIGURE 2: The velocity $v$ is plotted against the distance for different values of $\tau_{o}$ at $\alpha=0.1, \varepsilon=0.1, M=5, k=0.9$.

The flow rate, $q$, is given by

$$
\begin{aligned}
q & =\int_{0}^{1} u d y=\int_{0}^{1} \frac{\partial \psi}{\partial y} d y=\psi(1)-\psi(0) \\
& =\frac{-1}{\lambda_{1}\left(\tau_{0}+\left(1 / R_{e}\right)\right)}\left(1-\frac{\sinh \lambda_{1}}{\lambda_{1} \cosh \lambda_{1}}\right) \frac{\partial p_{0}}{\partial x} .
\end{aligned}
$$

The pressure rise is given by

$$
\Delta p_{0}=\int_{0}^{1} \frac{\partial p_{0}}{\partial x} d x=\frac{-\left(\tau_{0}+\left(1 / R_{e}\right)\right) \lambda_{1} q}{\left(1-\left(\sinh \lambda_{1} / \lambda_{1} \cosh \lambda_{1}\right)\right)} .
$$

Eliminating the pressure terms in (20) and (21), we have

$$
\begin{aligned}
\nabla^{2} \psi_{1 t} & +\psi_{0 y} \nabla^{2} \psi_{1 x}-\psi_{1 x} \nabla^{2} \psi_{0 y} \\
& =\left(\tau_{0}+\frac{1}{R_{e}}\right) \nabla^{4} \psi_{1}-\left(M+\frac{1}{K}\right) \nabla^{2} \psi_{1} .
\end{aligned}
$$

From conditions (24) and (25) we can write $\psi_{1}, \theta_{1}$, and $\phi_{1}$ in the form

$$
\begin{gathered}
\psi_{1}(x, y, t)=f(y) \cos \alpha(x-t)+g(y) \sin \alpha(x-t) . \\
\theta_{1}(x, y, t)=h_{1}(y) \cos \alpha(x-t), \\
\phi_{1}(x, y, t)=h_{2}(y) \cos \alpha(x-t) .
\end{gathered}
$$

Equation (28) can be simplified by using (29) and assuming that the wave number $\alpha=2 \pi d / \lambda$ is small, so the terms of $\alpha^{2}$ and higher can be neglected. 


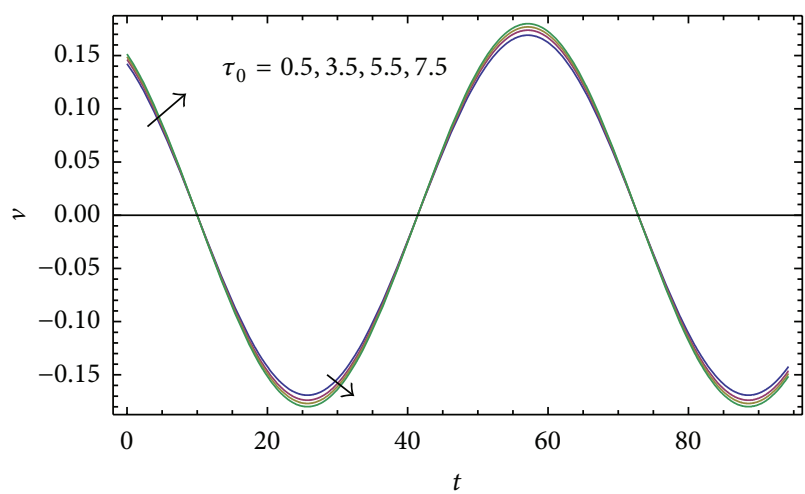

FIGURE 3: The velocity is plotted against the time for different values of $\tau_{o}$ at $\alpha=0.1, \varepsilon=0.1, M=5, k=0.9$.

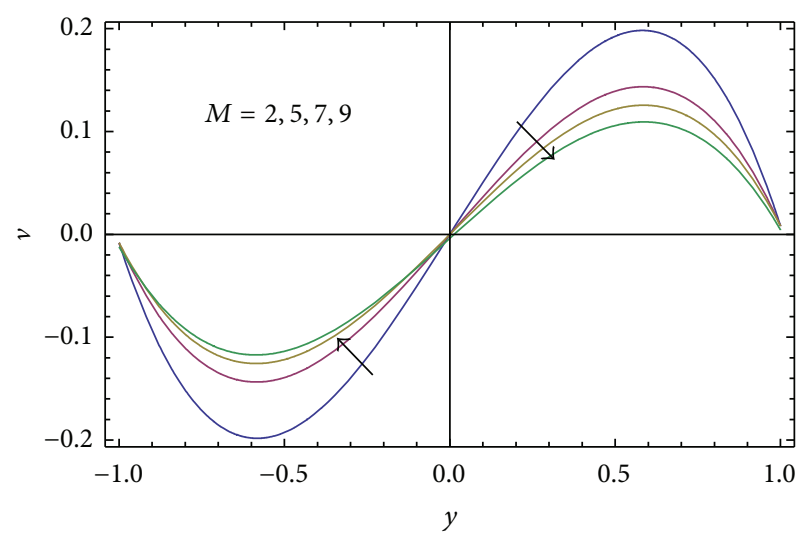

Figure 4: The velocity $v$ is plotted against the distance for different values of $M$ at $\alpha=0.1, \varepsilon=0.1, \tau_{o}=2, k=0.9$.

We get

$$
\begin{aligned}
\alpha\left[\left(\left(1-\frac{L}{\lambda_{1}}\right)+\frac{\cosh \lambda_{1} y}{\cosh \lambda_{1}}\right) f^{\prime \prime}\right. \\
\left.+L \frac{\cosh \lambda_{1} y}{\cosh \lambda_{1}} f\right] \sin \alpha(x-t) \\
-\alpha\left[\left(\left(1-\frac{L}{\lambda_{1}}\right)+\frac{\cosh \lambda_{1} y}{\cosh \lambda_{1}}\right) g^{\prime \prime}\right. \\
\left.\quad-L \frac{\cosh \lambda_{1} y}{\cosh \lambda_{1}} g\right] \cos \alpha(x-t) \\
=\left[\left(\tau_{0}+\frac{1}{R_{e}}\right) f^{\prime \prime \prime \prime}-\left(M+\frac{1}{R_{e}}\right) f^{\prime \prime}\right] \cos \alpha(x-t) \\
+\left[\left(\tau_{0}+\frac{1}{R_{e}}\right) g^{\prime \prime \prime \prime}-\left(M+\frac{1}{K}\right) g^{\prime \prime}\right] \sin \alpha(x-t) .
\end{aligned}
$$

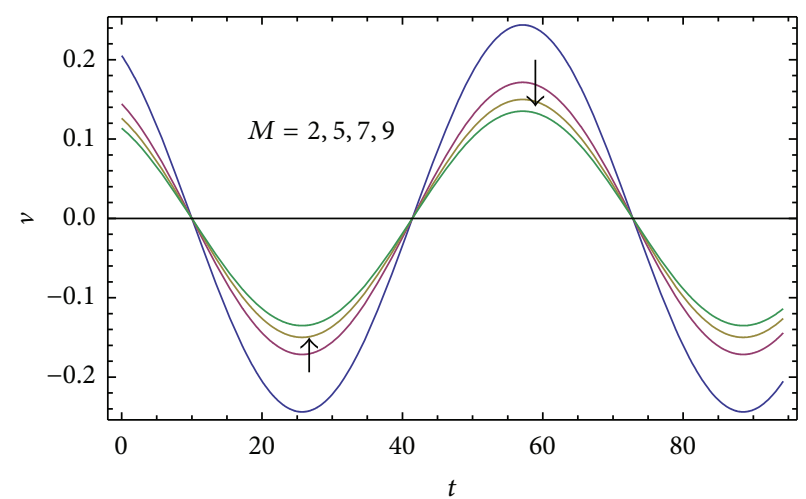

FIGURE 5: The velocity $v$ is plotted against the time $t$ for different values of $M$ at $\alpha=0.1, \varepsilon=0.1, \tau_{o}=2, k=0.9$.

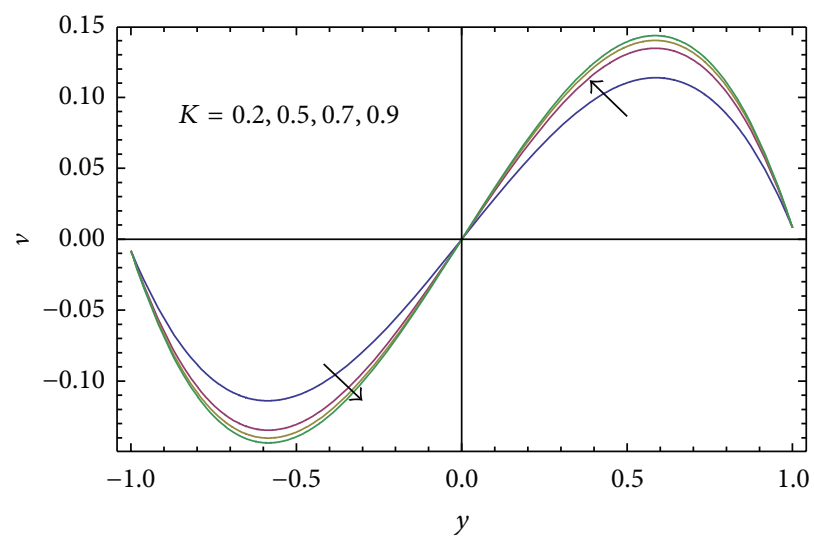

FIGURE 6: The velocity $v$ is plotted against the distance for different values of $k$ at $\alpha=0.1, \varepsilon=0.1, M=5, \tau_{o}=2$.

Collecting coefficients of $\cos \alpha(x-t)$ and $\sin \alpha(x-t)$ on either side of (30), two differential equations for $f(y)$ and $g(y)$ are obtained as follows:

$$
\begin{gathered}
-\alpha\left[\left(\left(1-\frac{L}{\lambda_{1}}\right)+\frac{\cosh \lambda_{1} y}{\cosh \lambda_{1}}\right) g^{\prime \prime}+L \frac{\cosh \lambda_{1} y}{\cosh \lambda_{1}} g\right] \\
=\left[\left(\tau_{0}+\frac{1}{R_{e}}\right) f^{\prime \prime \prime \prime}-\left(M+\frac{1}{K}\right) f^{\prime \prime}\right], \\
\alpha\left[\left(\left(1-\frac{L}{\lambda_{1}}\right)+\frac{\cosh \lambda_{1} y}{\cosh \lambda_{1}}\right) f^{\prime \prime}+L \frac{\cosh \lambda_{1} y}{\cosh \lambda_{1}} f\right] \\
=\left[\left(\tau_{0}+\frac{1}{R_{e}}\right) g^{\prime \prime \prime \prime}-\left(M+\frac{1}{K}\right) g^{\prime \prime}\right] .
\end{gathered}
$$

Equation (31) can be simplified by assuming that

$$
\begin{aligned}
& f(y)=f_{0}+\alpha f_{1}+\cdots, \\
& g(y)=g_{0}+\alpha g_{1}+\cdots .
\end{aligned}
$$




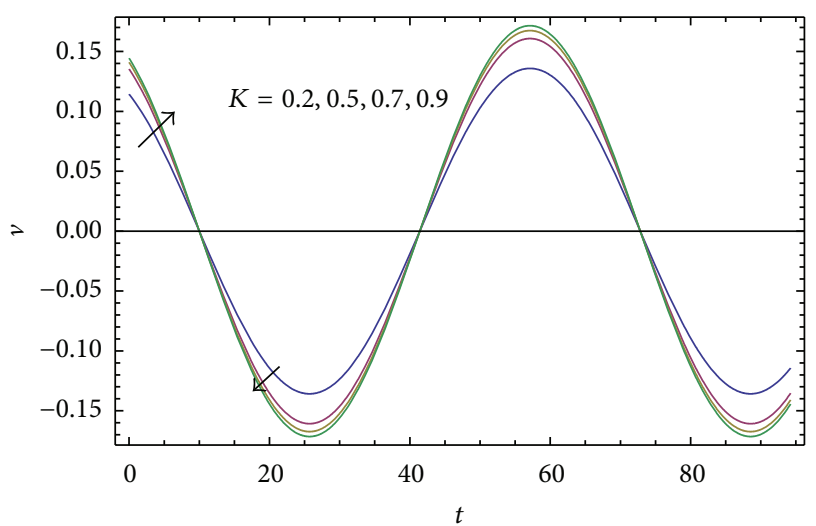

FIGURE 7: The velocity $v$ is plotted against the time $t$ for different values of $k$ at $\alpha=0.1, \varepsilon=0.1, M=5, \tau_{o}=2$.

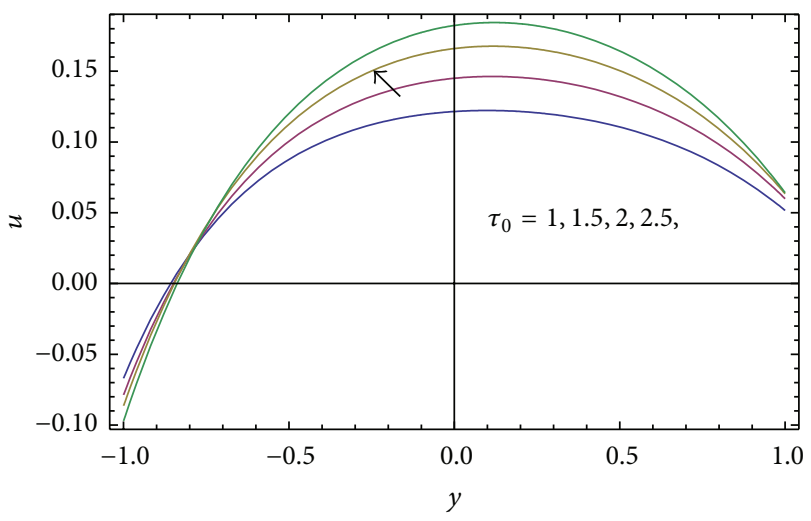

FIGURE 8: The velocity $u$ is plotted against the distance for different values of $\tau_{o}$ at $\alpha=0.1, \varepsilon=0.1, M=5, k=0.9$.

Substituting (32) into (31), and equating terms in $\alpha$, the following ordinary differential equations are obtained for $f_{o}$, $f_{1}, g_{0}$, and $g_{1}$, respectively:

$$
\begin{gathered}
f_{0}^{\prime \prime \prime \prime}-\lambda_{1}^{2} f_{0}^{\prime \prime}=0, \\
g_{0}^{\prime \prime \prime \prime}-\lambda_{1}^{2} g_{0}^{\prime \prime}=0, \\
-\left[\left(\left(1-\frac{L}{\lambda_{1}}\right)+\frac{\cosh \lambda_{1} y}{\cosh \lambda_{1}}\right) g_{0}^{\prime \prime}-L \frac{\cosh \lambda_{1} y}{\cosh \lambda_{1}} g_{0}\right] \\
=\left[\left(\tau_{0}+\frac{1}{R_{e}}\right) f_{1}^{\prime \prime \prime \prime}-\left(M+\frac{1}{K}\right) f_{1}^{\prime}\right], \\
{\left[\left(\left(1-\frac{L}{\lambda_{1}}\right)+\frac{\cosh \lambda_{1} y}{\cosh \lambda_{1}}\right) f_{0}^{\prime \prime}-L \frac{\cosh \lambda_{1} y}{\cosh \lambda_{1}} f_{0}\right]} \\
=\left[\left(\tau_{0}+\frac{1}{R_{e}}\right) g_{1}^{\prime \prime \prime \prime}-\left(M+\frac{1}{K}\right) g_{1}^{\prime \prime}\right]
\end{gathered}
$$

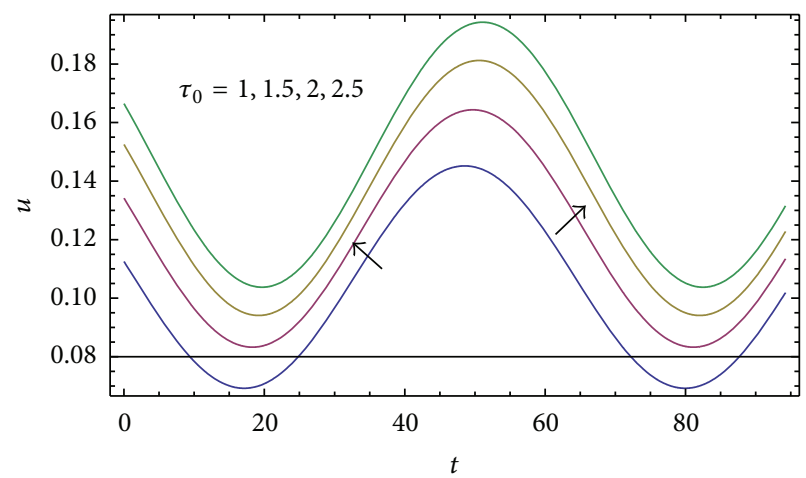

FIgURE 9: The velocity $u$ is plotted against the time $t$ for different values of $\tau_{o}$ at $\alpha=0.1, \varepsilon=0.1, M=5, k=0.9$.

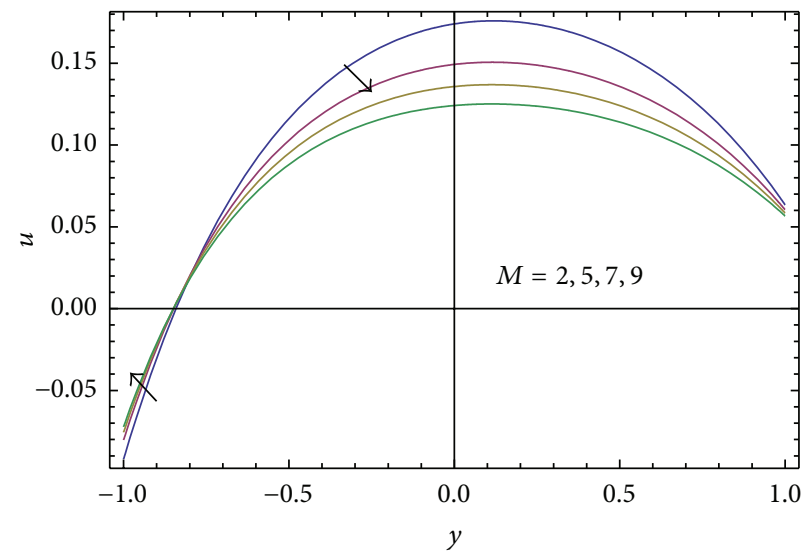

FIGURE 10: The velocity $u$ is plotted against the distance for different values of $M$ at $\alpha=0.1, \varepsilon=0.1, \tau_{o}=2, k=0.9$.

with boundary conditions:

$$
\begin{array}{cc}
f_{0}(1)=1, & f_{0}^{\prime}(1)=-A, \\
f_{0}(-1)=-1, & f_{0}^{\prime}(-1)=-A, \\
g_{0}(1)=0, & g_{0}^{\prime}(1)=0, \\
g_{0}(-1)=0, & g_{0}^{\prime}(-1)=0, \\
f_{1}(1)=0, & f_{1}^{\prime}(1)=0, \\
f_{1}(-1)=0, & f_{1}^{\prime}(-1)=0, \\
g_{1}(1)=0, & g_{1}^{\prime}(1)=0, \\
g_{1}^{\prime}(-1)=0, & g_{1}(-1)=0 .
\end{array}
$$




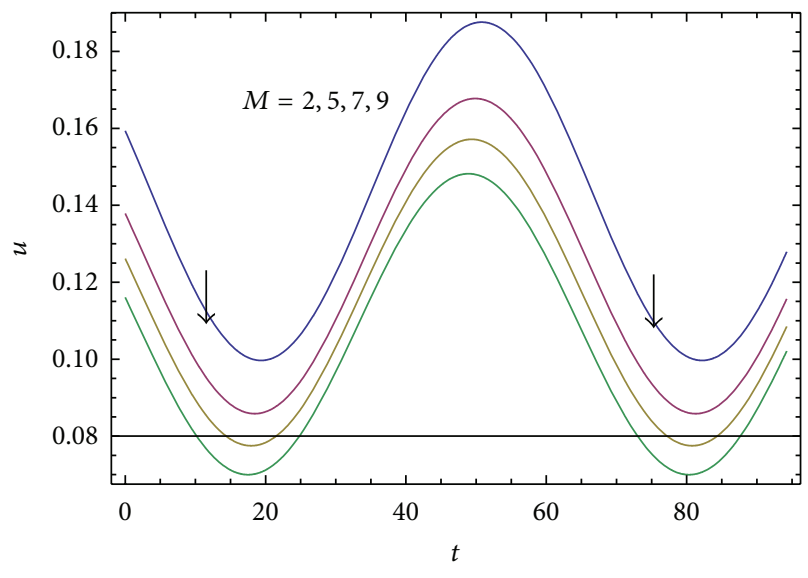

FIGURE 11: The velocity $u$ is plotted against the time $t$ for different values of $M$ at $\alpha=0.1, \varepsilon=0.1, \tau_{o}=2, k=0.9$.

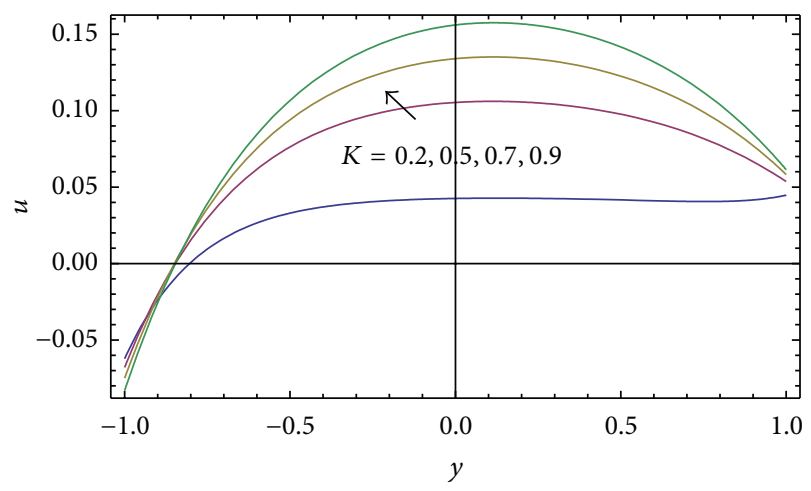

FIGURE 12: The velocity $u$ is plotted against the distance for different values of $k$ at $\alpha=0.1, \varepsilon=0.1, M=5, \tau_{o}=2$.

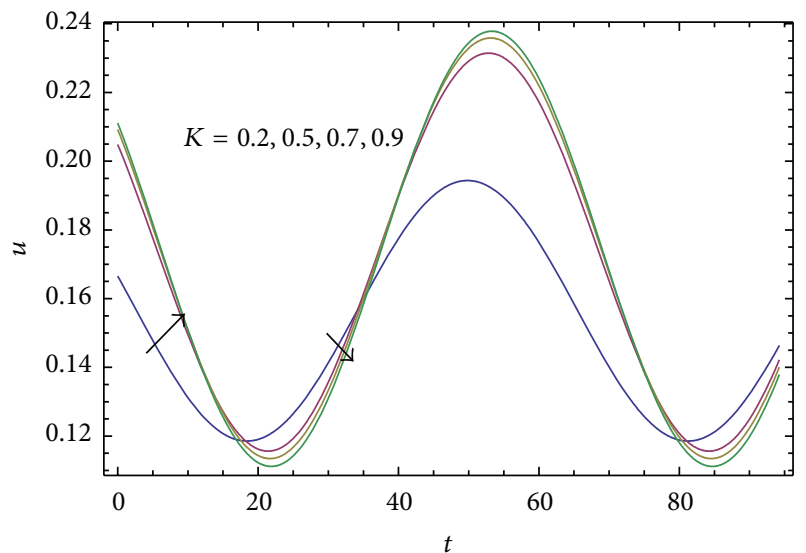

FIGURE 13: The velocity $u$ is plotted against the time $t$ for different values of $k$ at $\alpha=0.1, \varepsilon=0.1, M=5, \tau_{o}=2$.

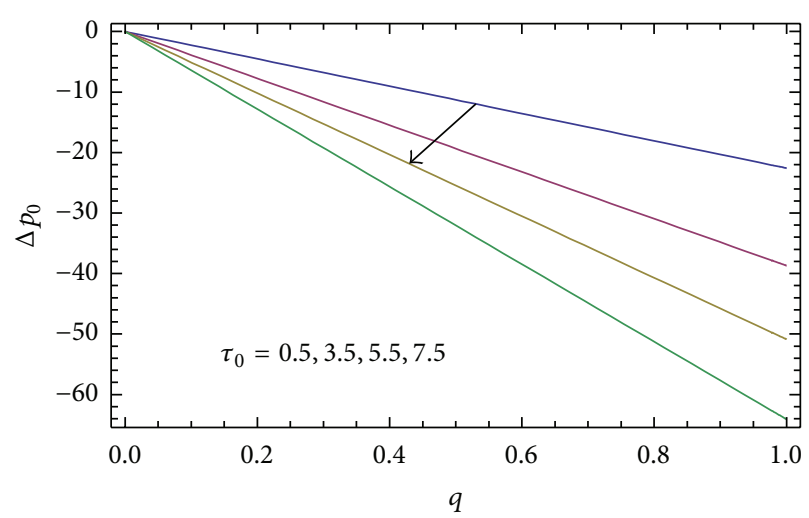

FIGURE 14: $\Delta p_{o}$ is plotted against $q$ for different values of $\tau_{o}$ at $\alpha=$ $0.1, \varepsilon=0.1, M=5, k=0.2$.

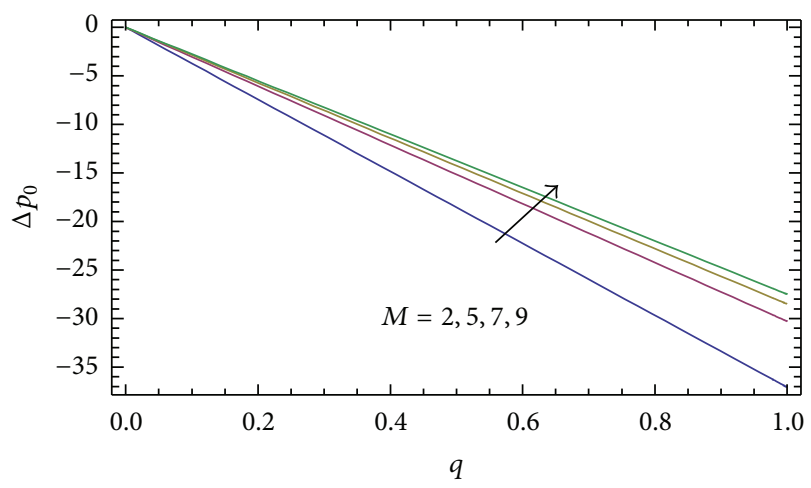

FIGURE 15: $\Delta p_{o}$ is plotted against $q$ for different values of $\tau_{o}$ at $\alpha=0.1$, $\varepsilon=0.1, M=5, k=0.2$.

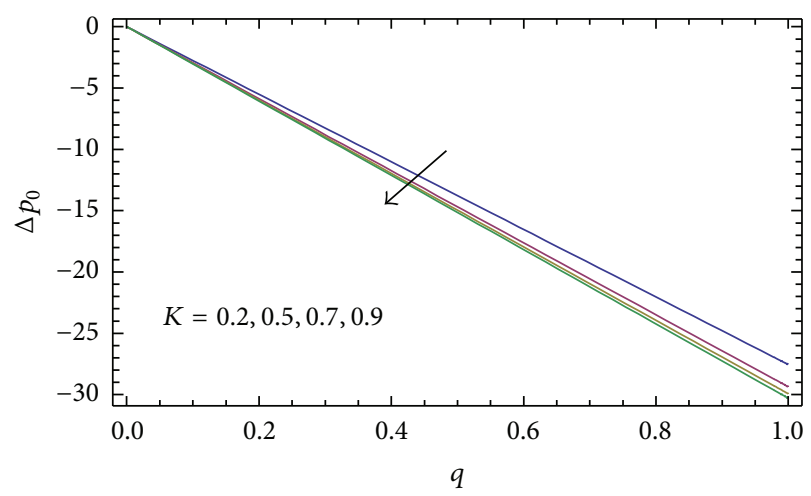

FIGURE 16: $\Delta p_{o}$ is plotted against $q$ for different values of $k$ at $\alpha=0.1$, $\varepsilon=0.1, M=5, \tau_{o}=2$. 


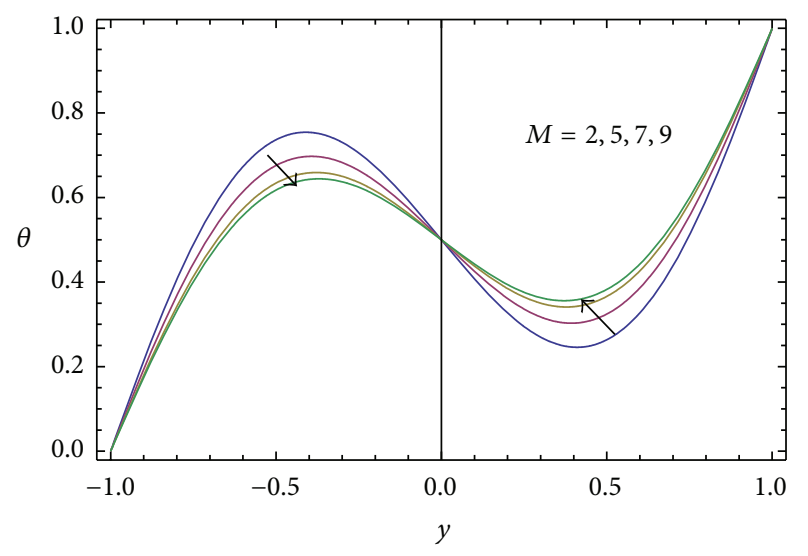

Figure 17: The temperature $\theta$ is plotted against the distance for different values of $M$ at $\alpha=0.1, \varepsilon=0.1, \tau_{o}=2, k=0.9$.

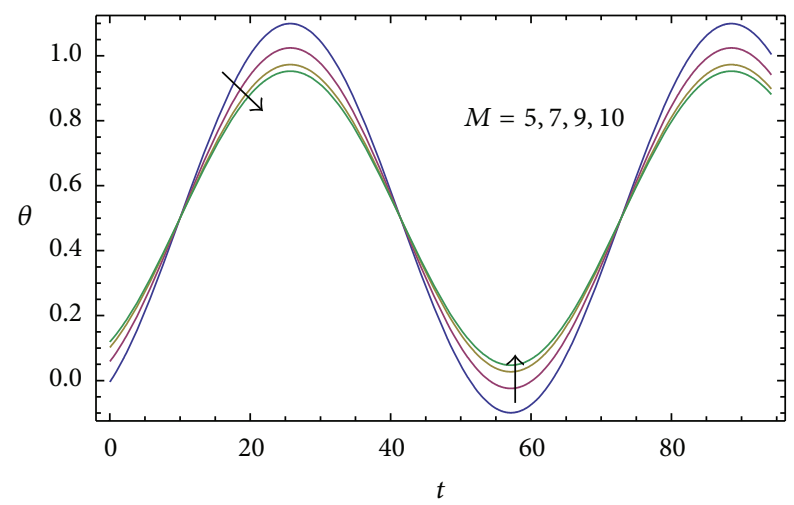

Figure 18: The temperature $\theta$ is plotted against the time $t$ for different values of $M$ at $\alpha=0.1, \varepsilon=0.1, \tau_{o}=2, k=0.9$.

Solving (33) by using (34), we get

$$
\begin{gathered}
f_{0}(y)=\lambda_{2} y-\lambda_{3} \sinh \lambda_{1} y, \\
f_{1}(y)=0, \quad g_{0}(y)=0, \\
g_{1}(y)=\lambda_{7} y \cosh \lambda_{1} y+\left(\lambda_{8}+\lambda_{9} y^{2}\right) \sinh \lambda_{1} y \\
+\lambda_{10} \sinh 2 \lambda_{1} y+\lambda_{11} y .
\end{gathered}
$$

Substituting (35) in (29), we get

$$
\begin{aligned}
\psi_{1}(x, y, t)= & \left(\lambda_{2} y-\lambda_{3} \sinh \lambda_{1} y\right) \cos \alpha(x-t) \\
+ & \alpha \sin \alpha(x-t) \\
& \times\left(\lambda_{7} y \cosh \lambda_{1} y+\left(\lambda_{8}+\lambda_{9} y^{2}\right) \sinh \lambda_{1} y\right. \\
& \left.+\lambda_{10} \sinh 2 \lambda_{1} y+\lambda_{11} y\right) .
\end{aligned}
$$

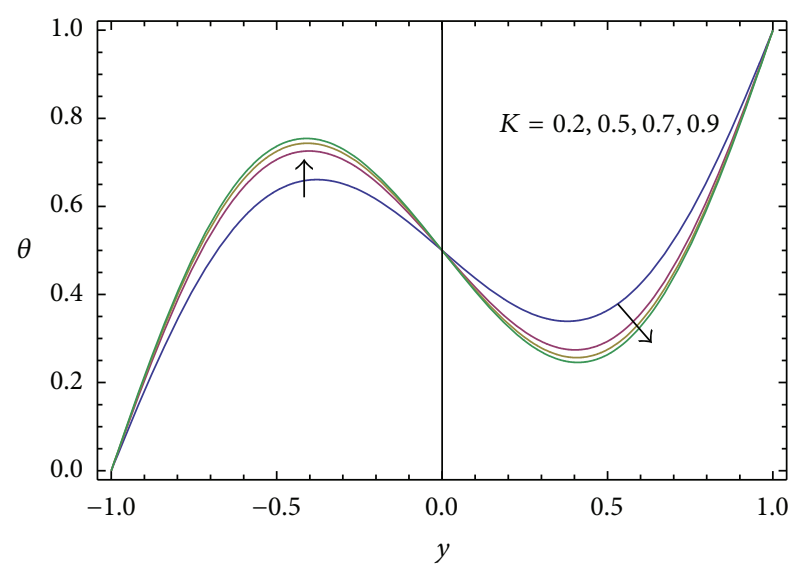

FIgURE 19: The temperature $\theta$ is plotted against the distance for different values of $k$ at $\alpha=0.1, \varepsilon=0.1, \tau_{o}=2, M=5$.

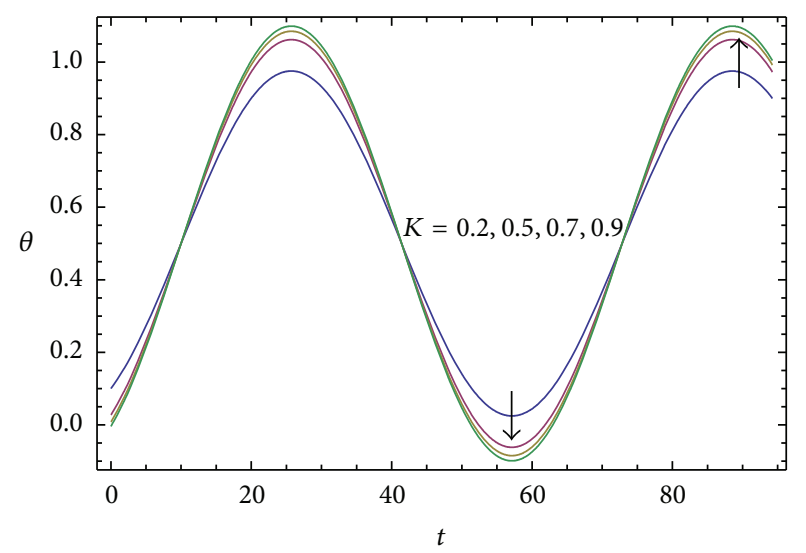

Figure 20: The temperature $\theta$ is plotted against the time $t$ for different values of $k$ at $\alpha=0.1, \varepsilon=0.1, \tau_{o}=2, M=5$.

Substituting (25) and (38) in (14), we get

$$
\begin{aligned}
& \psi(x, y, t)=\frac{-L}{\lambda_{1}}\left(y-\frac{\sinh \lambda_{1} y}{\lambda_{1} \cosh \lambda_{1}}\right) \\
&+\varepsilon\left(\left(\lambda_{2} y-\lambda_{3} \sinh \lambda_{1} y\right) \cos \alpha(x-t)\right. \\
&+ \alpha \sin \alpha(x-t) \\
& \times\left(\lambda_{7} y \cosh \lambda_{1} y+\left(\lambda_{8}+\lambda_{9} y^{2}\right) \sinh \lambda_{1} y\right. \\
&\left.\left.+\lambda_{10} \sinh 2 \lambda_{1} y+\lambda_{11} y\right)\right) .
\end{aligned}
$$




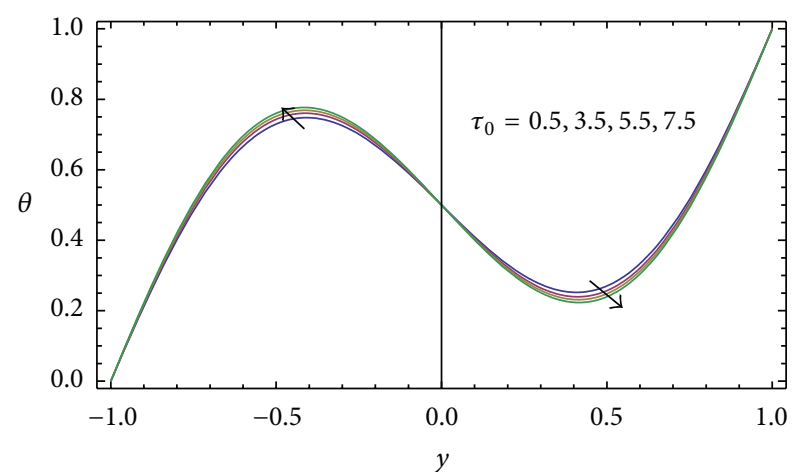

FIGURE 21: The temperature $\theta$ is plotted against the distance for different values of $\tau_{o}$ at $\alpha=0.1, \varepsilon=0.1, k=0.9, M=5$.

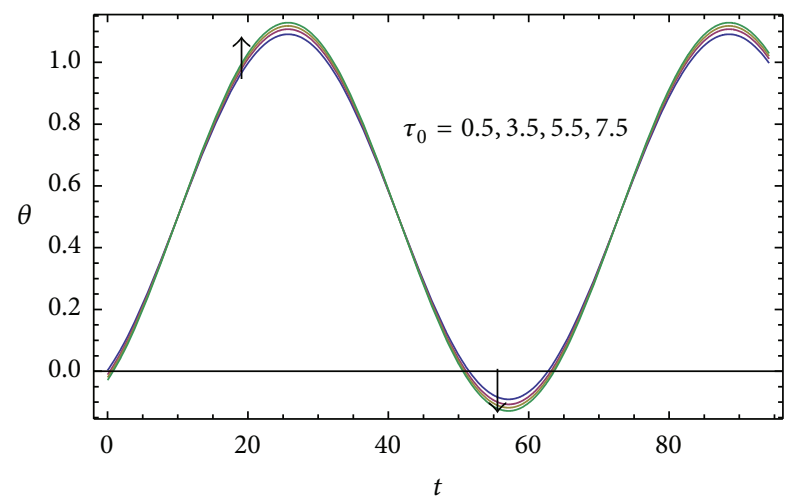

FIgURE 22: The temperature $\theta$ is plotted against the time $t$ for different values of $\tau_{o}$ at $\alpha=0.1, \varepsilon=0.1, k=0.9, M=5$.

The velocity components can be written as

$$
\begin{aligned}
& u(x, y, t)=\frac{-L}{\lambda_{1}}\left(1-\frac{\cosh \lambda_{1} y}{\cosh \lambda_{1}}\right) \\
& +\varepsilon\left(\left(\lambda_{2}-\lambda_{3} \lambda_{1} \cosh \lambda_{1} y\right) \cos \alpha(x-t)\right. \\
& +\alpha \sin \alpha(x-t) \\
& \times\left(\lambda_{7} \cosh \lambda_{1} y+\lambda_{1} \lambda_{7} y \sinh \lambda_{1} y\right. \\
& \quad+\lambda_{1}\left(\lambda_{8}+\lambda_{9} y^{2}\right) \cosh \lambda_{1} y \\
& \quad+2 \lambda_{1} \lambda_{10} \cosh 2 \lambda_{1} y \\
& \left.\left.\quad+\lambda_{11}+2 \lambda_{9} y \sinh \lambda_{1} y\right)\right) \\
& v(x, y, t)=\alpha \varepsilon\left[\left(\lambda_{2} y-\lambda_{3} \sinh \lambda_{1} y\right) \sin \alpha(x-t)\right]
\end{aligned}
$$

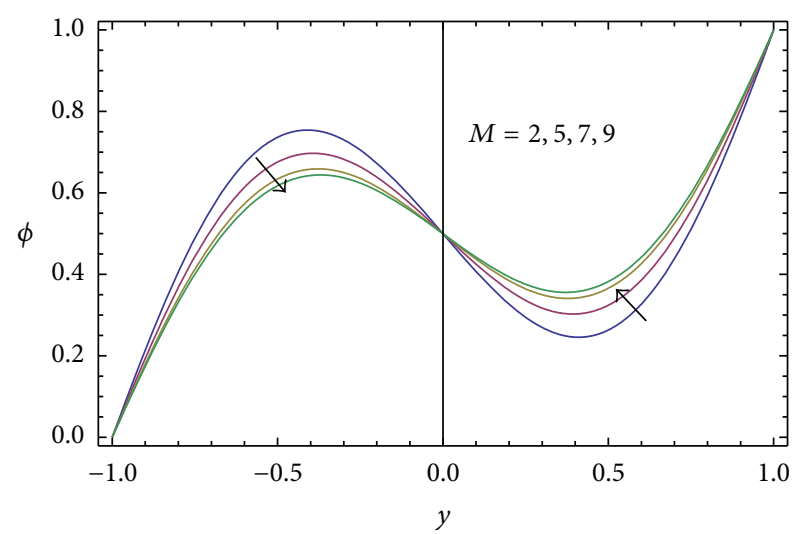

FIGURE 23: The concentration distribution $\phi$ is plotted against the distance for different values of $M$ at $\alpha=0.1, \varepsilon=0.1, \tau_{o}=2, k=0.9$.

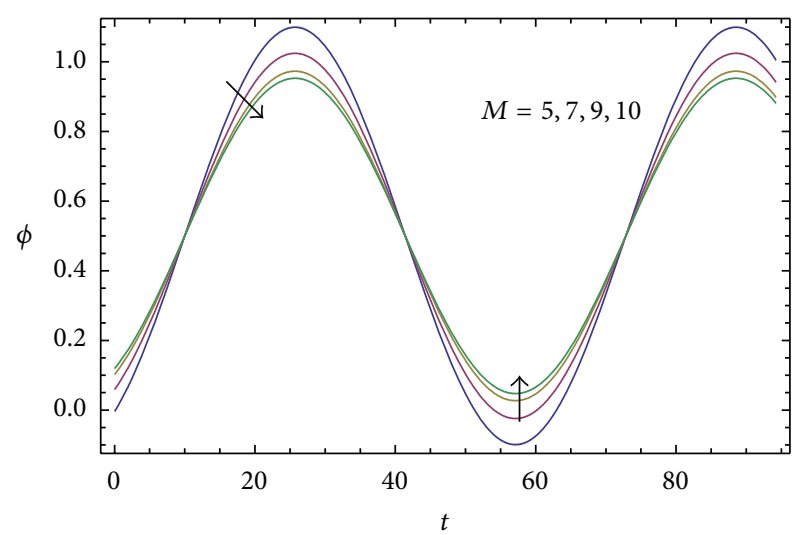

FIgURE 24: The concentration distribution $\phi$ is plotted against the time $t$ for different values of $M$ at $\alpha=0.1, \varepsilon=0.1, \tau_{o}=2, K=0.9$.

Substituting (39) in (17), (18), (22), and (23) and using (24) we get

$$
\begin{gathered}
\theta_{o}(y)=\frac{y+1}{2}, \quad \varphi_{o}(y)=\frac{y+1}{2} \\
\theta_{1}(x, y, t)=\left(\left(-\lambda_{1}^{2} \lambda_{12} y+\lambda_{1}^{2} \lambda_{12} y^{3}+6 \lambda_{13} y \sinh \lambda_{1}\right.\right. \\
\left.\left.-6 \lambda_{13} \sinh \lambda_{1} y\right)\left(6 \lambda_{1}^{2}\right)^{-1}\right) \\
\quad \times \cos \alpha(x-t) \\
\varphi_{1}(x, y, t)=\left(\left(-\lambda_{1}^{2} \lambda_{14} y+\lambda_{1}^{2} \lambda_{12} y^{3}+6 \lambda_{15} y \sinh \lambda_{1}\right.\right. \\
\left.\left.-6 \lambda_{15} \sinh \lambda_{1} y\right)\left(6 \lambda_{1}^{2}\right)^{-1}\right) \cos \alpha(x-t) .
\end{gathered}
$$




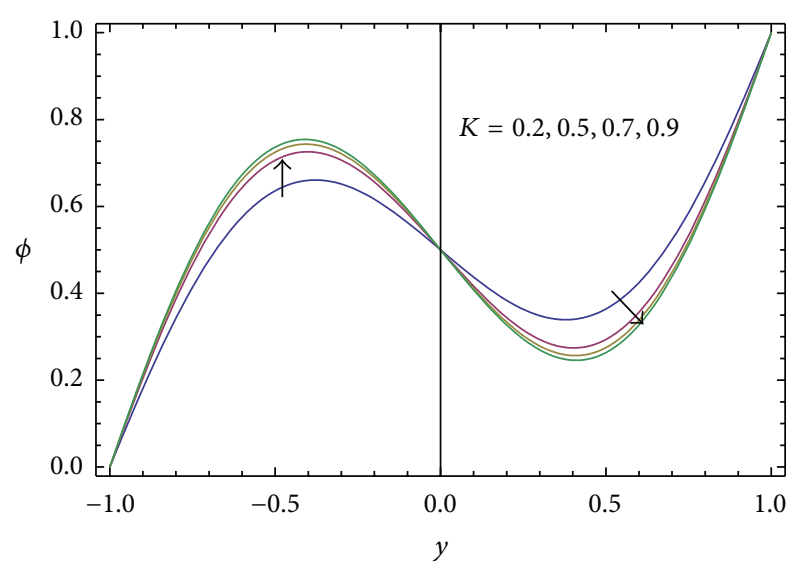

FIgURE 25: The concentration distribution $\phi$ is plotted against the distance for different values of $k$ at $\alpha=0.1, \varepsilon=0.1, \tau_{o}=2, M=5$.

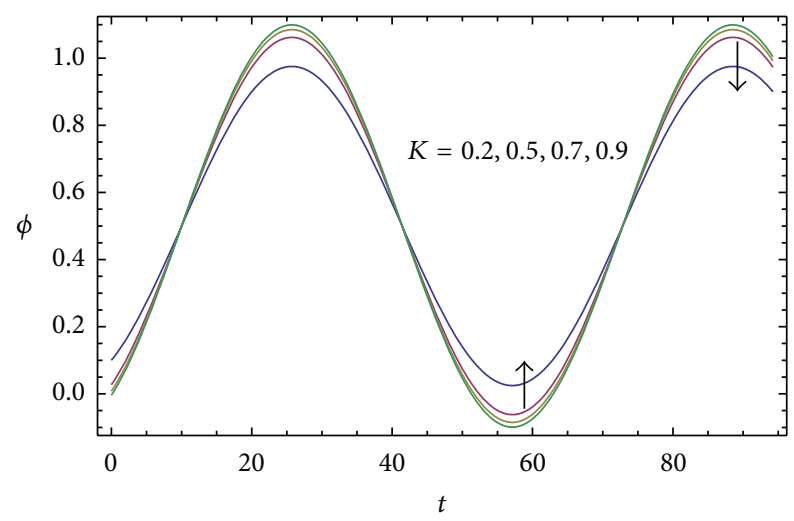

Figure 26: The concentration distribution $\phi$ is plotted against the time $t$ for different values of $k$ at $\alpha=0.1, \varepsilon=0.1, \tau_{o}=2, M=5$.

Substituting (41) in (14), we get

$\theta(x, y, t)$

$$
\begin{aligned}
& =\left(\frac{y+1}{2}\right) \\
& +\varepsilon \alpha\left(\left(-\lambda_{1}^{2} \lambda_{12} y+\lambda_{1}^{2} \lambda_{12} y^{3}\right.\right. \\
& \left.\left.\quad+6 \lambda_{13} y \sinh \lambda_{1}-6 \lambda_{13} \sinh \lambda_{1} y\right)\left(6 \lambda_{1}^{2}\right)^{-1}\right) \\
& \quad \times \cos \alpha(x-t),
\end{aligned}
$$

$$
\phi(x, y, t)
$$

$$
=\frac{y+1}{2}+\varepsilon \alpha\left(\left(-\lambda_{1}^{2} \lambda_{14} y+\lambda_{1}^{2} \lambda_{14} y^{3}\right.\right.
$$

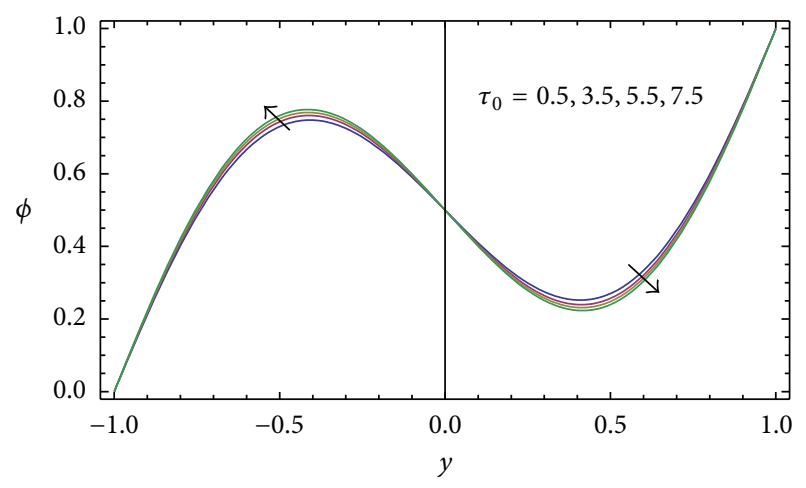

FIGURE 27: The concentration distribution $\phi$ is plotted against the distance for different values of $\tau_{o}$ at $\alpha=0.1, \varepsilon=0.1, M=5, k=0.9$.

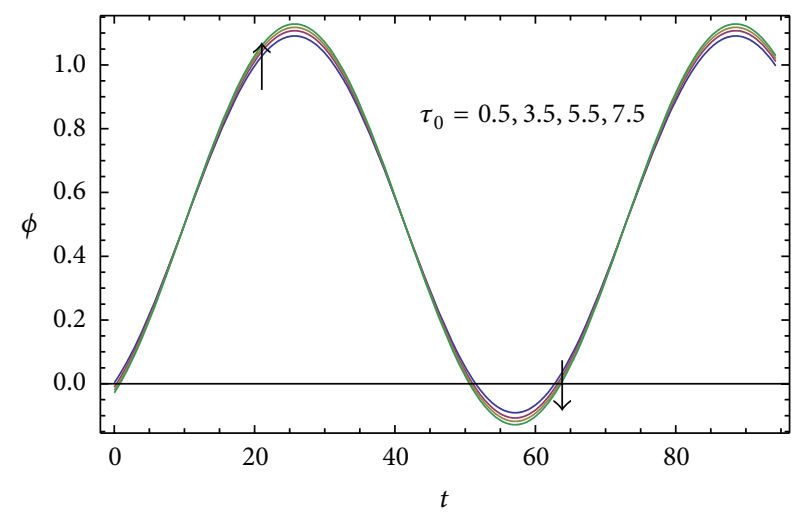

FIgURE 28: The concentration distribution $\phi$ is plotted against the time $t$ for different values of $\tau_{0}$ at $\alpha=0.1, \varepsilon=0.1, K=0.9, M=5$.

$$
\begin{aligned}
& \left.+6 \lambda_{15} y \sinh \lambda_{1}-6 \lambda_{15} \sinh \lambda_{1} y\right) \\
& \left.\times\left(6 \lambda_{1}^{2}\right)^{-1}\right) \cos \alpha(x-t),
\end{aligned}
$$

where

$$
\begin{aligned}
& A=\psi_{0 y y}(1)=\frac{L \sinh \lambda_{1}}{\lambda_{1} \cosh \lambda_{1}}, \\
& \lambda_{2}=\frac{A \sinh \lambda_{1}+\lambda_{1} \cosh \lambda_{1}}{-\sinh \lambda_{1}+\lambda_{1} \cosh \lambda_{1}}, \\
& \lambda_{3}=\frac{1+A}{-\sinh \lambda_{1}+\lambda_{1} \cosh \lambda_{1}}, \\
& \lambda_{4}=\frac{\lambda_{1}^{2} \lambda_{3}\left(1-\left(L / \lambda_{1}\right)\right)}{\left(\tau_{0}+\left(1 / R_{e}\right)\right)}, \quad \lambda_{6}=\frac{L \lambda_{2}}{\cosh \lambda_{1}},
\end{aligned}
$$




$$
\begin{aligned}
& \lambda_{7}=\left(\left(24 \lambda_{1} \lambda_{4}+60 \lambda_{6}\right) \sinh \lambda_{1}\right. \\
& \left.-\left(24 \lambda_{1}^{2} \lambda_{4}+60 \lambda_{1} \lambda_{6}\right) \cosh \lambda_{1}\right) \\
& \times\left(48 \lambda_{1}^{4}\left(\lambda_{1} \cosh \lambda_{1}-\sinh \lambda_{1}\right)\right)^{-1}, \\
& \lambda_{8}=\left(\left(24 \lambda_{1}^{2} \lambda_{4}+48 \lambda_{1} \lambda_{6}\right) \sinh \lambda_{1}\right. \\
& -12 \lambda_{1}^{2} \lambda_{6} \cosh \lambda_{1}-2 \lambda_{5} \sinh 2 \lambda_{1} \\
& \left.+4 \lambda_{1} \lambda_{5} \cosh 2 \lambda_{1}\right) \\
& \times\left(48 \lambda_{1}^{4}\left(\lambda_{1} \cosh \lambda_{1}-\sinh \lambda_{1}\right)\right)^{-1}, \\
& \lambda_{9}=\frac{\left(12 \lambda_{1}^{2} \lambda_{6}\right) \cosh \lambda_{1}-12 \lambda_{1} \lambda_{6} \sinh \lambda_{1}}{48 \lambda_{1}^{4}\left(\lambda_{1} \cosh \lambda_{1}-\sinh \lambda_{1}\right)}, \\
& \lambda_{10}=\frac{\left(2 \lambda_{5}\right) \sinh \lambda_{1}-2 \lambda_{1} \lambda_{5} \cosh \lambda_{1}}{48 \lambda_{1}^{4}\left(\lambda_{1} \cosh \lambda_{1}-\sinh \lambda_{1}\right)} \\
& \lambda_{11}=\left(\left(24 \lambda_{1}^{2} \lambda_{4}\right)-12 \lambda_{1} \lambda_{5} \sinh 2 \lambda_{1}+3 \lambda_{1} \lambda_{5} \sinh \lambda_{1}\right. \\
& -\lambda_{1} \lambda_{5} \sinh 3 \lambda_{1}+48 \lambda_{1} \lambda_{6} \\
& \left.+\left(12 \lambda_{1} \lambda_{6}-30 \lambda_{6}\right) \cosh 2 \lambda_{1}\right) \\
& \times\left(48 \lambda_{1}^{4}\left(\lambda_{1} \cosh \lambda_{1}-\sinh \lambda_{1}\right)\right)^{-1}, \\
& \lambda_{12}=\frac{\lambda_{2} \tan \alpha(x-t)}{C_{p}}, \quad \lambda_{13}=\frac{\lambda_{3} \tan \alpha(x-t)}{C_{p}}, \\
& \lambda_{14}=\frac{\lambda_{2} \sin \alpha(x-t)}{D_{m} \cos \alpha(x-t)}, \quad \lambda_{15}=\frac{\lambda_{3} \sin \alpha(x-t)}{D_{m} \cos \alpha(x-t)} \text {. }
\end{aligned}
$$

\section{Results and Discussion}

In this work, we have studied the effect of different parameters of the considered problem on the solutions of the momentum, heat, and mass equations. This discussion is illustrated graphically through a set of Figures 2-28. Since Figures 2 and 3 illustrated the influence of the Casson parameter $\tau_{0}$ on the velocity component $v$, hence we noticed that the velocity component $v$ increases with the increase of the Casson parameter $\tau_{0}$ for $0 \leq y \leq 1$ and decreases for $-1 \leq$ $y \leq 0$. The effect of the magnetic parameter $M$ on the velocity component $v$ is shown in Figures 4 and 5. These figures reveal that the velocity component $v$ decreases with the increase of $M$ at $0 \leq y \leq 1$ and increases at $-1 \leq y \leq 0$. Figures 6 and 7 depicted the behavior of permeability parameter $K$ on the velocity component $v$. It is noticed that the velocity component $v$ decreases with $K$ in the region $-1 \leq y \leq 0$, and it increases in the region $0 \leq y \leq 1$. Figures 8 and 9 showed the effect of $\tau_{0}$ on the velocity component $u$; it is clear that the velocity component $u$ increases with increasing of $\tau_{0}$. Also, the velocity component $u$ decreases when the magnetic parameter $M$ increases, then shown through Figures 10 and 11. From Figures 12 and 13, since the motion is sinusoidal, we have seen that the longitudinal velocity $u$ increases or decreases as the permeability parameter $K$ increases. Figures 14 and 16 illustrated the influence of $\tau_{0}$ and $K$ on the pressure rise $\Delta p_{0}$. These figures show that the pressure rise $\Delta p_{0}$ decreases with the increase of both $\tau_{0}$ and $K$. Figure 15 displayed the effected of the magnetic parameter $M$ on the pressure rise $\Delta p_{0}$; it is noticed that the magnitude of $\Delta p_{0}$ increases with $M$. We can see from Figures 17 and 18 that the temperature $\theta$ decreases when $M$ increases in the interval $-1 \leq y \leq 0$, while it increases in the interval $0 \leq y \leq 1$. We observed from Figures 19, 20, 21, and 22 that the temperature $\theta$ increases when $K$ and $\tau_{0}$ increase in the interval $-1 \leq y \leq 0$, and it decreases in the interval $0 \leq y \leq 1$. In Figures 23 and 24 , it is seen that the concentration distribution $\phi$ decreases with $M$ in the region $-1 \leq y \leq 0$, but it increases in the region $0 \leq y \leq 1$. The concentration distribution $\phi$ increases when $K$ and $\tau_{0}$ increase in the interval $-1 \leq y \leq 0$, and it decreases in the interval $0 \leq y \leq 1$, this is shown in Figures 25, 26, 27, and 28 .

\section{Conflict of Interests}

The authors declare that there is no conflict of interests regarding the publication of this paper.

\section{References}

[1] P. Muthu, B. V. R. Kumar, and P. Chandra, "On the influence of wall properties in the peristaltic motion of micropolar fluid," ANZIAM Journal, vol. 45, no. 2, pp. 245-260, 2003.

[2] Y. C. Fung, C. S. Yih, and J. Asme, "Peristaltic transport," Journal of Applied Mechanics, vol. 35, no. 4, pp. 669-675, 1968.

[3] J. C. Burns and T. Parkes, "Peristaltic motion," Journal of Fluid Mechanics, vol. 29, pp. 731-743, 1970.

[4] A. V. Mernone and J. N. Mazumdar, "Biomathematical modelling of physiological fluids using a Casson fluid with emphasis to peristalsis," Australasian Physical and Engineering Sciences in Medicine, vol. 23, no. 3, pp. 94-100, 2000.

[5] A. V. Mernone, J. N. Mazumdar, and S. K. Lucas, "A mathematical study of peristaltic transport of a Casson fluid," Mathematical and Computer Modelling, vol. 35, no. 7-8, pp. 895912, 2002.

[6] Kh. S. Mekheimer and T. H. Al-Arabi, "Nonlinear peristaltic transport of MHD flow through a porous medium," International Journal of Mathematics and Mathematical Sciences, vol. 2003, no. 26, pp. 1663-1682, 2003.

[7] Kh. S. Mekheimer, "Non-linear peristaltic transport of magnetohydrodynamic flow in an inclined planar channel," Arabian Journal for Science and Engineering A, vol. 28, no. 2, pp. 183-201, 2003.

[8] P. Nagarani and G. Sarojamma, "Peristaltic transport of a Casson fluid in an asymmetric channel," Australasian Physical and Engineering Sciences in Medicine, vol. 27, no. 2, pp. 49-59, 2004.

[9] P. Nagarani, "Peristaltic transport of a casson fluid in an inclined channel," Korea Australia Rheology Journal, vol. 22, no. 2, pp. 105-111, 2010.

[10] E. F. Elshehawey, N. T. Eldabe, E. M. Elghazy, and A. Ebaid, "Peristaltic transport in an asymmetric channel through a 
porous medium," Applied Mathematics and Computation, vol. 182, no. 1, pp. 140-150, 2006.

[11] R. Bharagava, H. S. Takhar, S. Rawat, A. Tasveer Beg, and O. Anwar Beg, "finite element solutions for non-Newtonian pulsatile flow in a non Darican porous medium conduit," Nonlinear Analysis Modeling and Control, vol. 12, no. 3, pp. 317327, 2007.

[12] Kh. S. Mekheimer and Y. Abd elmaboud, "The influence of heat transfer and magnetic field on peristaltic transport of a Newtonian fluid in a vertical annulus: application of an endoscope," Physics Letters A: General, Atomic and Solid State Physics, vol. 372, no. 10, pp. 1657-1665, 2008.

[13] S. Nadeem, N. S. Akbar, N. Bibi, and S. Ashiq, "Influence of heat and mass transfer on peristaltic flow of a third order fluid in a diverging tube," Communications in Nonlinear Science and Numerical Simulation, vol. 15, no. 10, pp. 2916-2931, 2010.

[14] Y. Abd elmaboud and K. S. Mekheimer, "Non-linear peristaltic transport of a second-order fluid through a porous medium," Applied Mathematical Modelling, vol. 35, no. 6, pp. 2695-2710, 2011.

[15] Y. Abd Elmaboud, "Thermomicropolar fluid flowin a porous channel with peristalsis," Journal of Porous Media, vol. 14, no. 11, pp. 1033-1045, 2011.

[16] N. T. El-dabe, S. N. Sallam, M. A. Mohamed, M. Y. Abo Zaid, and A. Abd-Emonem, "Magnetohydrodynamic peristaltic motion with heat and mass transfer of a Jeffery fluid in a tube through porous medium," Innovative System Design and Engineering, vol. 2, no. 4, 2011.

[17] M. Mustafa, S. Hina, T. Hayat, and A. Alsaedi, "Influence of wall properties on the peristaltic flow of a nanofluid: Analytic and numerical solutions," International Journal of Heat and Mass Transfer, vol. 55, no. 17-18, pp. 4871-4877, 2012.

[18] O. Anwr Beg and D. Tripathi, "Mathematica simulation of peristaltic pumoing with double-diffusive convection in nanofluids: a bio-nano-engineering model," The Journal of Nanoengineering and Nanosystems, 2012.

[19] N. T. El-dabe, S. M. Elshaboury, A. A. Hasan, and M. A. Elogail, "MHD Peristaltic flow of a couple stress fluids with heat and mass transfer through a porous medium," Innovative System Design and Engineering, vol. 3, no. 5, 2012.

[20] N. T. El-dabe, K. A. Kamel, M. Galila abd-Allah, and S. F. Ramadan, "Heat absorption and chemical reaction effects on Peristaltic motion of micropolar fluid through a porous medium in the presence of magnetic field," The African Journal of Mathematics and Computer Science Research, vol. 6, no. 5, pp. 94-101, 2013.

[21] A. Ebaid and H. Emad Aly, "Exact analytical solution of the peristaltic nanofluids flow in an asymmetric channel with flexible walls and slip condition: application to cancer treatment," Computational and Mathematical Methods in Medicine, vol. 2013, Article ID 825376, 8 pages, 2013.

[22] H. Emad and A. Ebaid, "New analytical and numerical solutions for mixed convection boundary- layer nanofluid flow along an inclined plate embedded in porous medium," Journal of Applied Mathematics, vol. 2013, Article ID 219486, 7 pages, 2013.

[23] Y. Abd Elmaboud, "Unsteady flow of magneto thermomicropolar fluid in a porous channel with peristalsis: unsteady separation," Journal of Heat Transfer-Transactions of the ASME, vol. 135, no. 7, Article ID 072602, 2013.

[24] S. Noreen, T. Hayat, A. Alsaedi, and Qasim, "Mixed convection heat and mass transfer in perisltic flow," Indian Journal of Physics, vol. 87, no. 9, pp. 889-896, 2013.
[25] T. Hayat, S. Noreen, and M. Qasim, "Influence of heat and mass transfer on the peristaltic transport of a phan-thien-tanner fluid," Zeitschrift fur Naturforschung A, vol. 68, pp. 751-758, 2013.

[26] S. Noreen, "Correction: mixed convection peristaltic flow of third order nanofluid with an induced magnetic field," PLoS ONE, vol. 8, no. 12, 2013.

[27] M. Nakamura and T. Sawada, "Numerical study on the flow of a non-Newtonian fluid through an axisymmetric stenosis," Journal of Biomechanical Engineering, vol. 110, no. 2, pp. 137-143, 1988. 

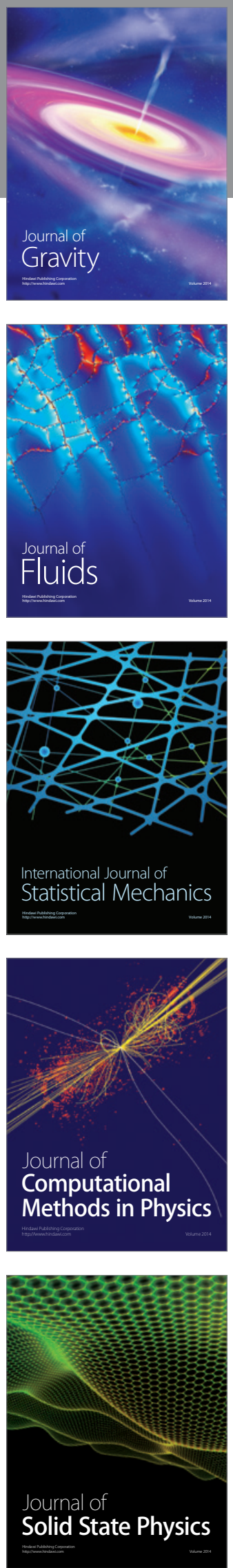

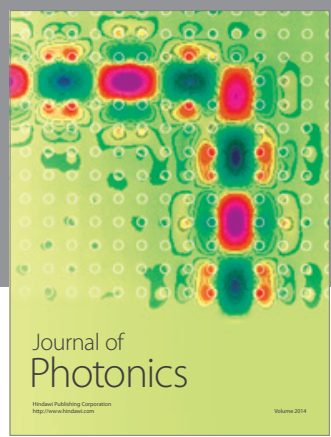

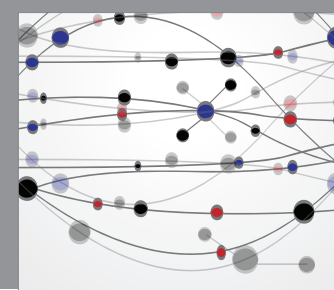

The Scientific World Journal

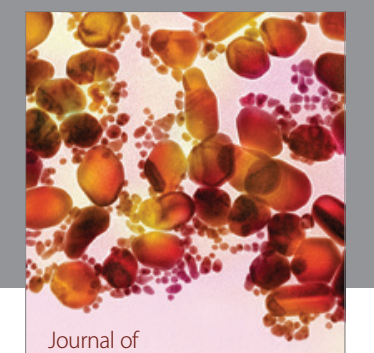

Soft Matter
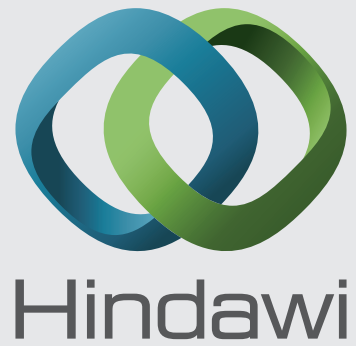

Submit your manuscripts at

http://www.hindawi.com
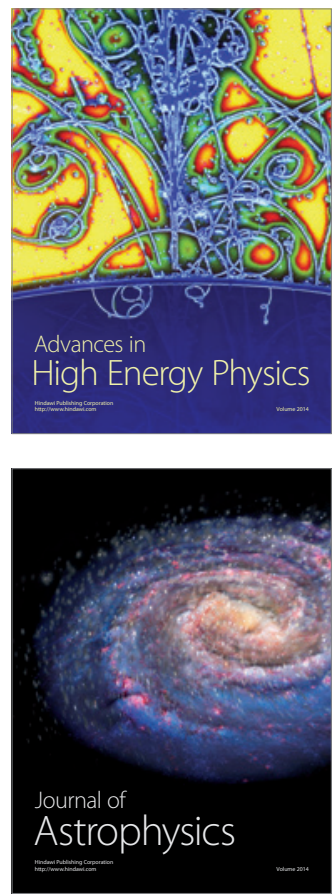
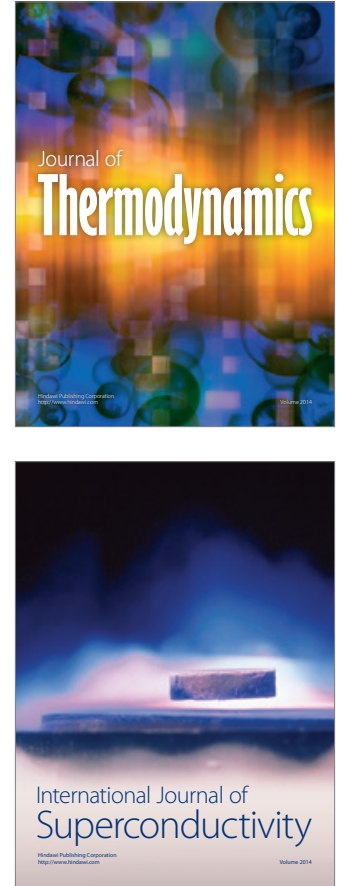
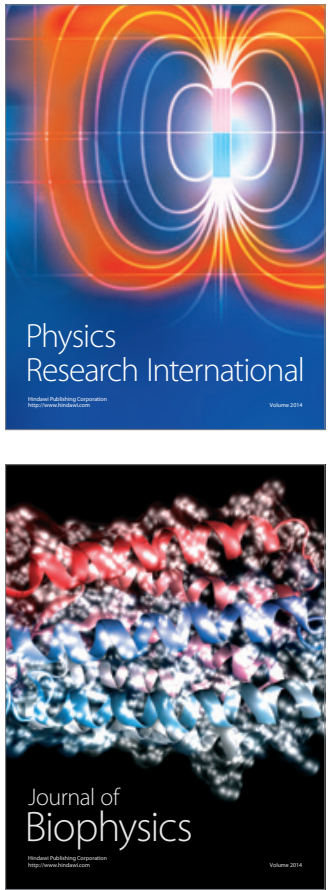
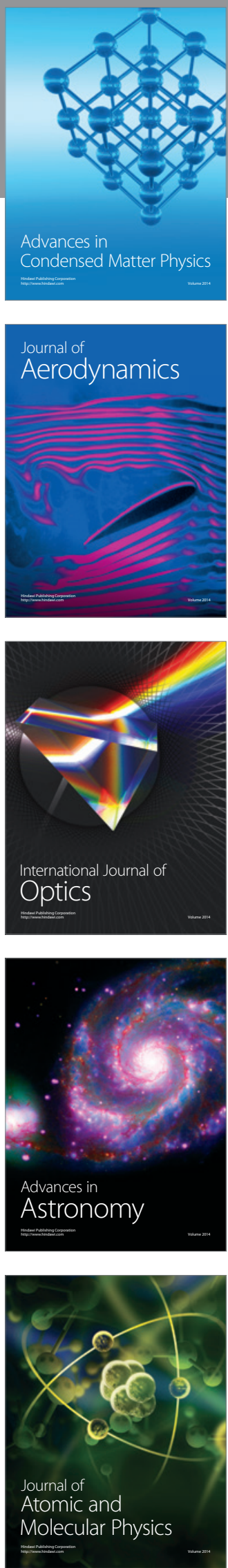